\title{
Großer oder kleiner Unterschied? Geschlechtsspezifische Betrachtung ist wichtig
}

Dass es geschlechtsspezifische Besonderheiten auch bei psychischen Erkrankungen gibt, wussten schon die Gründer der Medizin, wie Hippokrates oder Aretaeos von Kappadokien. Allerdings waren es in erster Linie postnatale Psychosen und Depressionen, zyklusabhängige oder andere im Zusammenhang mit reproduktiven Vorgängen auftretende psychische Störungen bei Frauen, die zunächst Interesse fanden. Erst mit der allgemeinen Sensibilität für geschlechtsspezifische Fragen etwa ab Mitte der siebziger Jahre des letzten Jahrhunderts begann auch in der Psychiatrie eine Ära, in der diese Aspekte eine Rolle spielten.

In der psychiatrischen klinischen Realität waren die ersten frauenspezifischen Behandlungskonzepte mit der Etablierung von „Mutter-Kind-Einrichtungen“ verbunden. Auf psychiatrischen Tagungen und Kongressen hielten geschlechtsspezifische Aspekte zunächst über vereinzelte Vorträge und Symposien Einzug. Mittlerweile nimmt das Interesse für „GenderAspekte" in allen Bereichen der Psychiatrie und Psychotherapie ständig zu, was sich auch in der Gründung eines DGPPN-Referats „Frauen- und geschlechtsspezifische Fragen in der Psychiatrie“ 2001 zeigte; mittlerweile als „Gender-Referat" bezeichnet.

Eine Bestandsaufnahme des psychiatrischen und psychotherapeutischen Wissens aus der geschlechtsspezifischen Perspektive im kürzlich herausgegebenen Handbuch „Geschlechtsspezifische Psychiatrie und Psychotherapie“
(Hrsg. A. Rohde, A. Marneros) machte deutlich, dass die geschlechtsspezifische Betrachtung in den verschiedenen Bereichen unterschiedlich weit fortgeschritten ist und dass die Differenzen in klinischem Bild und Verlauf ebenso wie die daraus resultierenden Behandlungserfordernisse bei den einzelnen Störungen sehr unterschiedlich sein können.

Eines aber wird immer klarer: Unabhängig vom Ausmaß der Unterschiedlichkeit ist die geschlechtsspezifische Betrachtung von Bedeutung - um klinisches Bild und Verlauf zu verstehen, aber insbesondere auch um weitere Erkenntnisse zur Genese von Störungen zu gewinnen.

In diesem Kontext stehen im vorliegenden Heft die Beiträge zur neuropsychologischen Forschung (K. Wietasch) und zur funktionellen Bildgebung (D. Braus). F. Pillmann beschäftigt sich in seinem Beitrag mit einem seltenen Psychosetyp, der eine deutliche Geschlechtsspezifität aufweist - den akuten vorübergehenden Psychosen. Und schließlich wird in zwei Beiträgen die spezielle Betreuung von psychisch kranken Frauen im Kontext von Geburt und Postpartalzeit dargestellt.

Unabhängig davon, ob großer oder kleiner Unterschied und unabhängig vom Kontext die geschlechtsspezifische Betrachtung nimmt in fast allen Bereichen der psychiatrischen Wissenschaft zu; eine immer selbstverständlichere Einbeziehung dieser Aspekte auch im klinischen Alltag sollte das Ziel sein.

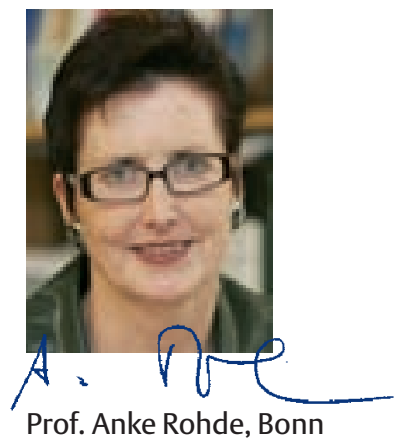

